\title{
0171 OUTCOMES FOLLOWING MAJOR TRAUMA: MEASURING RECOVERY POSTDISCHARGE
}

B J Gabbe, ${ }^{*}$ A M Sutherland, A P Hannaford, P A Cameron Correspondence: Department of Medicine, Nursing and Health Sciences, School of Public Health and Preventive Medicine Monash University The Alfred Hospital Commercial Rd Melbourne, Victoria 3004, Australia

10.1136/ip.2010.029215.171

Background The Victorian State Trauma Registry (VSTR) collects outcomes of major trauma survivors at 6-months postinjury but the need for longer term outcomes data for monitoring recovery and quantifying the burden of injury is recognised.

Aims Describe the outcomes of patients at 6,12,18 and 24 months following major trauma, and determine key time points for follow-up.

Methods All 692 VSTR blunt major trauma patients, surviving to discharge from the Level 1 trauma services (October 2006 to March 2007) were followed-up at 6,12, 18 and 24 months postinjury by telephone. SF-12, Extended Glasgow Outcome Scale (GOS-E), pain scores and measures of work-related disability were collected.

Results The follow-up rates were $86 \%, 82 \%, 81 \%$ and $83 \%$ at $6,12,18$ and 24-months postinjury, respectively. Improvement in outcomes was evident over time, although the rate of improvement from 18 to 24 -months was small. $21 \%$ of cases had fully recovered (GOS-E $=8$ ) at 24 -months. $68 \%$ had returned to work but only $57 \%$ of these cases had returned to their preinjury workplace. $24 \%$ of patients reported persistent moderate-severe pain, and median physical and mental health scores remained below population norms at 24 -months.

Discussion and Conclusion Follow-up at 24 months postinjury is feasible and the follow-up rate is comparable to 6 and 12 -months after injury. Disability was prevalent, highlighting the burden of injury borne by this group even 2 -years after injury. 